\title{
Lésions ostéo-fibreuses des maxillaires: discordance entre diagnostics radiologique et histologique
}

\author{
Hind Koumiti, Sophia Haitami, Mokrane Khazana, Ihsane Ben Yahya \\ Faculté de Médecine dentaire, rue Abou Alaa Zahr, Casablanca, Maroc \\ hindkoumiti@live.fr
}

Les lésions ostéo-fibreuses sont des entités relativement peu fréquentes, regroupant des lésions bénignes caractérisées par le remplacement de la matrice osseuse normale par un tissu ostéo-fibreux.

Elles sont uni ou multifocales à croissance lente, de découverte fortuite et portent des dénominations diverses. Lorsqu'elles impliquent un nombre restreint de dents et qu'elles se situent dans la partie antérieure, elles sont décrites en tant que dysplasie osseuse péri-apicale. Une lésion analogue à localisation postérieure est décrite en tant que dysplasie osseuse focale. Lorsqu'elles sont étendues et apparaissent dans plusieurs quadrants, elles sont connues sous terme de dysplasie osseuse floride.

Les lésions ostéo-fibreuses regroupent plusieurs lésions dont les aspects radiologique et histologique sont très proches. Le diagnostic histologique est souvent difficile, nécessitant la connaissance du tableau clinique et radiologique.

Une patiente âgée de 56 ans a consulté en janvier 2008 pour une fistule apparue après l'extraction de la 36. Le bilan radiologique, comprenant une radiographie panoramique et un examen tomodensitométrique, montre une image mixte diffuse dans chaque branche horizontale de la mandibule. Une biopsie dans la région de la 36 fait évoquer une ostéite.

En 2010, la patiente consulte pour des douleurs mandibulaires diffuses. Un bilan radiologique est réalisé et il révèle la présence d'une image mixte diffuse dans les branches horizontales de la mandibule. Sous anesthésie locale, plusieurs prélèvements ont été effectués et les examens anatompathologiques ont conclu à des diagnostics différents.

Pour le prélèvement effectué dans la région molaire gauche, l'examen anatomo-pathologique a conclu à un odontome complexe, pour celui effectué dans la région molaire droite à une ostéite chronique, et pour celui effectué dans la région prémolaire droite a conclu à une ostéite chronique associée à un cémentome.

Devant ces différents diagnostics, les lames ont été relues et les diagnostics confirmés. 